'Instituto Milenio para la Investigación en Depresión y Personalidad (MIDAP). Santiago, Chile.

2Escuela de Psicología, Pontificia Universidad Católica de Chile. Santiago, Chile.

${ }^{3}$ Psicomedica, Clinical \& Research Group. Santiago, Chile.

${ }^{4}$ Departamento de Psiquiatría y Salud Mental, Hospital Clínico Universidad de Chile. Santiago, Chile. ${ }^{5}$ Mood Disorders Program, Tufts Medical Center. Boston, MA, USA. ${ }^{6}$ Department of Psychiatry, Tufts University School of Medicine. Boston, MA, USA.

${ }^{7}$ Escuela de Psicología, Facultad de Humanidades, Universidad de Santiago de Chile. Santiago, Chile.

${ }^{8}$ CITIAPS, Universidad de Santiago de Chile. Santiago, Chile.

${ }^{9}$ Núcleo Milenio para Mejorar la Salud Mental de Adolescentes y Jóvenes (Imhay). Santiago, Chile.

${ }^{10} \mathrm{Centro}$ de Psicoterapia de Premisas. San Luis Potosí, México.

"Departamento de Psiquiatría y Salud Mental Campus Oriente, Facultad de Medicina, Universidad de Chile. Santiago, Chile. apsicólogo, Doctor en Psicología Clínica. bPsicólogo, Doctor en Psicología. 'Psicóloga, Magíster en Psicología Clínica.

Fuente de apoyo financiero: Este estudio recibió apoyo del Fondo de Innovación para la Competitividad (FIC) del Ministerio de Economía, Fomento y Turismo, a través de la Iniciativa Científica Milenio, Proyecto IS130005. Los autores declaran no tener conflictos de interés.

Recibido el 20 de diciembre de 2019 , aceptado el 18 de marzo de 2020

Correspondencia a: Sergio Gloger

Av. Salvador 149, Of. 1101. Santiago, Chile.

sergio.gloger@psicomedica.cl

\section{Validación de la versión en español del Childhood Trauma Questionnaire-Short Form en Chile, en una muestra de pacientes con depresión clínica}

\author{
ALEX BEHN $^{1,2, a}$, PAUL A. VÖHRINGER ${ }^{1,3,4,5,6}$, \\ PABLO MARTÍNEZ $Z^{1,3,4,7,8,9, \mathrm{~b}}$, ANA PAULA DOMÍNGUEZ ${ }^{10, \mathrm{c}}$, \\ ARANTZA GONZÁLEZ ${ }^{3}$, MARÍA I. CARRASCO ${ }^{3}$, \\ SERGIO GLOGER ${ }^{1,3,11}$
}

\section{Validation of the Childhood Trauma Questionnaire-Short Form in Chile}

Background: Childhood Trauma Questionnaire-Short Form (CTQ-SF) is an instrument to assess child abuse and neglect Aim: to adapt and confirm the psychometric properties of the Spanish version of the CTQ-SF in Chile. Material and Methods: The CTQ-SF was applied to 89 clinically depressed subjects (77.5\% women) who consulted at an outpatient mental health clinic. Confirmatory factor analysis (CFA), reliability (Cronbach's $\alpha$ ) tests, and convergent validity analyses with clinical markers of complex depression were carried out. Results: The Chilean version of the CTQ-SF demonstrated an acceptable fit to a five-factor model, with adequate psychometric properties. The CFA revealed that a better fit to a five-factor model would be achieved after elimination of two items from the physical neglect scale, the less reliable scale of the questionnaire. The physical abuse scale discriminated between patients with a complex depression versus non-complex depression, and all the CTQ-SF's scales discriminated between patients with high suicide risk and/or history of psychiatric admissions versus those patients without this background. Conclusions: the Chilean version of the CTQ-SF shows evidence of structural and discriminant validity, and reliability, in a clinical sample. Better alternatives to specifically assess the physical neglect construct should be developed.

(Rev Med Chile 2020; 148: 336-343)

Key words: Child Abuse; Depressive Disorder; Psychological Tests; Validation Study.
L a evidencia científica ha demostrado de manera robusta que el estrés adverso temprano (EAT), en la forma del abuso y negligencia infantil, es común y tiene efectos neurobiológicos negativos y persistentes ${ }^{1}$. Un estudio epidemiológico pionero en San Diego, Estados Unidos (i.e., ACEs study), indicó que $52,1 \%$ de los partici- pantes habían sufrido alguna forma de EAT en la infancia ${ }^{2}$; mientras que, en Chile, un estudio efectuado en atención primaria de salud halló este antecedente en $82 \%$ de pacientes deprimidos ${ }^{3}$. La exposición a EAT se ha relacionado consistentemente con una mayor incidencia de psicopatología (siendo la depresión una de las más estudiadas) 
y enfermedades físicas, tales como enfermedades cardiovasculares, cáncer, enfermedad pulmonar obstructiva crónica y diabetes ${ }^{1,4}$. Por estos motivos, mediante su estrecha vinculación a resultados adversos en salud, la contribución del EAT a la carga de enfermedad global es sustantiva ${ }^{4}$.

Considerando que la detección oportuna de la exposición a EAT es un primer paso crítico para reducir o evitar las graves consecuencias en la vida adulta asociadas al trauma temprano ${ }^{5,6}$, es de suma relevancia la disponibilidad de instrumentos confiables y válidos para la evaluación del EAT en poblaciones clínicas y no clínicas ${ }^{7}$. Entre los distintos instrumentos para este propósito, revisados sistemáticamente por Saini et al. ${ }^{7}$, el Childhood Trauma Questionnaire Short-Form (CTQ-SF) destaca por ser el más estudiado y por tener adecuadas propiedades psicométricas. Este instrumento, desarrollado por Bernstein et al. ${ }^{8}$, no se limita a la evaluación exclusiva del abuso sexual y físico sino a la pesquisa breve, auto informada, retrospectiva (i.e., en la edad adulta), no intrusiva y exhaustiva del abuso emocional, físico, sexual, y de la negligencia emocional y física ${ }^{9}$, cumpliendo con los requerimientos para una indagación cuidadosa en trauma temprano ${ }^{10} \mathrm{y}$ demostrando amplia utilidad clínica e investigativa en varios países ${ }^{7}$.

La versión española del CTQ-SF, validada inicialmente en una muestra de 185 mujeres que recibían tratamiento ambulatorio u hospitalario para trastornos de personalidad y/o distimia ${ }^{11}$, cuenta con propiedades psicométricas adecuadas, replicando los hallazgos de estudios previos ${ }^{8,10}$. No obstante, resultados previos ${ }^{9,11}$, destacan la necesidad de contar con estudios comparativos y adaptaciones culturales, en grupos poblacionales diferentes (p.ej., ambos sexos, para Hernández et al. $)^{11}$, para contar con métodos fiables para pesquisar EAT. Más aún, el antecedente de EAT es común y se asocia a una clínica más compleja de la depresión ${ }^{3}$, motivo por el que su exploración en la práctica clínica habitual y su consideración en las orientaciones técnicas para el tratamiento de la patología debiese ser fundamental ${ }^{6}$. El presente estudio tuvo por objetivo adaptar y confirmar en Chile las propiedades psicométricas de la versión española del CTQ-SF, para su validación en una muestra de pacientes con depresión clínica que consultan en un servicio de salud mental ambulatorio (Psicomedica, Clinical \& Research Group) en Santiago.

\section{Material y Método}

Estudio de tipo cuantitativo, observacional, correlacional y transversal, para la validación de una escala para la pesquisa de EAT, efectuado en un servicio de salud mental ambulatorio en Santiago, Chile.

\section{Participantes}

Se incluyó a consultantes que tuvieran al menos 18 años de edad, con capacidad de lecto-escritura y con confirmación diagnóstica de depresión según criterios diagnósticos CIE-10. Se excluyeron pacientes con enfermedad bipolar confirmada. No hubo otros criterios de inclusión/exclusión. Participaron 89 individuos $(77,5 \%$ mujeres, $\mathrm{n}=69)$, con una media de 39,2 años $(\mathrm{DE}=13,9)$, que consultaron en el servicio de salud mental ambulatorio del estudio, durante el período de estudio (2017-2018). En la Tabla 1, se observa que, entre sus características clínicas, destacan el antecedente de depresiones previas/recurrentes $(57,3 \%, \mathrm{n}=51)$, la historia familiar de trastornos del ánimo $(53,9 \%, \mathrm{n}=48)$ y el diagnóstico de depresión moderada $(47,2 \%, \mathrm{n}=42)$.

\section{Procedimientos}

Se efectuó un muestreo sistemático y por conveniencia, en el que se invitó al estudio a todos los pacientes de 18 años o más que asistieron

\section{Tabla 1. Características demográficas y clínicas de la muestra $(n=89)$}

\begin{tabular}{|lr|}
\hline Variable & \multicolumn{1}{c|}{ Datos } \\
\hline Edad, años & $39,2(13,9)$ \\
\hline Sexo, mujer & $69(77,5)$ \\
\hline Depresión previa/recurrente & $51(57,3)$ \\
Historia familiar de trastornos del ánimo & $48(53,9)$ \\
\hline Antecedentes de hospitalizaciones psiquiátricas & $11(12,4)$ \\
Alto riesgo suicida (MINI) & $12(13,5)$ \\
$\begin{array}{l}\text { Diagnóstico de depresión } \\
\text { Leve }\end{array}$ & $8(9,0)$ \\
$\quad \begin{array}{l}\text { Moderada } \\
\text { Grave } \\
\text { Con psicosis, alto riesgo suicida o } \\
\text { refractariedad }\end{array}$ & $42(47,2)$ \\
\hline
\end{tabular}

Nota. Los datos son media (desviación estándar) o frecuencia (porcentaje). MINI = Mini Entrevista Neuropsiquiátrica Internacional. 
al servicio de salud mental ambulatorio para la confirmación de su diagnóstico de depresión. El 93\% de los invitados al estudio aceptó participar. Previo al ingreso a su cita médica para la confirmación de su diagnóstico de depresión, se obtuvo su consentimiento informado por escrito y se les hizo entrega de la adaptación chilena del CTQ-SF para el auto informe de EAT. Con posterioridad, en el contexto de la cita médica, las entrevistas clínicas diagnósticas de estos pacientes fueron efectuadas por médico psiquiatra, o por un médico general con entrenamiento en salud mental debidamente supervisado, informado de los objetivos del estudio. Finalmente, los datos registrados durante la cita médica en las fichas clínicas de los pacientes fueron revisados por miembros del equipo de investigación, quienes utilizaron un formulario estandarizado para orientar la extracción de esta información. Este estudio y su consentimiento informado fueron aprobados por el Comité de Seguridad de la Investigación Científica de la Pontificia Universidad Católica de Chile y por el Comité de Ética de Psicomedica, Clinical \& Research Group.

\section{Instrumentos}

Childhood Trauma Questionnaire-Short Form (CTQ-SF). Escala de auto reporte de 28 ítems, para adultos y adolescentes, que evalúa exhaustiva y retrospectivamente el antecedente de $\mathrm{EAT}^{8}$, basado en la versión extendida de 70 ítems (CTQ) ${ }^{12}$. Compuesto de 5 subescalas que evalúan abuso emocional (AE), abuso físico (AF), abuso sexual (AS), negligencia emocional (NE) y negligencia física (NF), cada una representada en 5 ítems evaluados con respuestas de escala tipo Likert de 5 puntos (desde "nunca" hasta "casi siempre"). Cuenta con tres ítems adicionales para la detección de respuestas socialmente aceptadas o subregistro de EAT. La estructura factorial de 5 dimensiones, reportada por Bernstein et al. ${ }^{12}$, ha sido consistentemente replicada en otros estudios ${ }^{9-11,13}$. Los coeficientes $\alpha$ de Cronbach para las escalas de la versión española del CTQ-SF fueron: $\operatorname{AE~}(0,87)$, AF $(0,89)$, AS $(0,94)$, NE $(0,83)$ y NF $(0,66)^{11}$. En el presente estudio se realizaron adaptaciones lingüísticas a los ítems 3, 4, 19, y 24, para facilitar su comprensión por la muestra chilena, sin alterar la carga semántica de los mismos, siendo esto último comprobado por jueces expertos en el tema. La adaptación chilena del CTQ-SF se presenta en un documento suplementario.
Marcadores de complejidad clínica de la depresión. Los siguientes marcadores de complejidad clínica de la depresión fueron extraídos de la ficha clínica de cada paciente en el servicio de salud mental ambulatorio del estudio: 1) depresión compleja; 2) alto riesgo suicida; $\mathrm{y}, 3$ ) antecedentes de hospitalizaciones psiquiátricas. Cabe destacar que esta información fue obtenida en el marco de entrevistas clínicas diagnósticas para la confirmación del diagnóstico de depresión en los pacientes.

Depresión compleja. Bajo la denominación de depresión compleja se han agrupado las categorías depresión grave, depresión con psicosis, alto riesgo suicida, o refractariedad a tratamiento, $y$ que corresponden a los niveles de severidad que requieren un mayor uso de recursos, en comparación a los niveles leves y moderados, según lo consignado por la Guía Clínica de Depresión como por la canasta de prestaciones GES. ${ }^{(14-15)}$ Asimismo, esta definición es similar a la "depresión compleja y grave" del National Institute for Health and Care Excellence (NICE), que incluye a pacientes cuya presentación clínica implica riesgo vital o funcional grave, con elevados costos para los servicios de salud. ${ }^{(16)}$ El diagnóstico de depresión se efectuó en base a CIE-10, y se utilizaron los criterios operativos de la Guía Clínica de Depresión para la determinación de la gravedad. ${ }^{(14)}$

Alto riesgo suicida. Pacientes que obtuvieron 10 o más puntos en el módulo C "Riesgo de Suicidio" de la Mini Entrevista Neuropsiquiátrica Internacional (MINI), versión 5.0.0 en español, ${ }^{(17)}$ que permite la puntuación del riesgo de suicidio a través de 6 preguntas (escala de 0 a 33 puntos). Corresponde a una evaluación independiente y complementaria a la recomendada por la Guía Clínica de Depresión y las orientaciones del Programa Nacional de Prevención del Suicidio. ${ }^{(14,18)} \mathrm{La}$ MINI es una entrevista diagnóstica estructurada, válida, confiable, y de breve duración, basada en los criterios diagnóstico DSM-IV y CIE-10. ${ }^{(17)}$

Antecedentes de hospitalizaciones psiquiátricas. Presencia o ausencia de hospitalizaciones psiquiátricas previas, según información entregada por el paciente durante su evaluación clínica.

\section{Análisis de los datos}

Se condujeron análisis exploratorios para la caracterización de la muestra y del instrumento. Se efectuó un análisis factorial confirmatorio (CFA) 
para comprobar la validez estructural del modelo de cinco dimensiones propuesto originalmente para el CTQ-SF${ }^{8}$. Debido a la distribución asimétrica de los datos se ajustó un modelo estructural con el método de máxima verosimilitud con la técnica de corrección robusta de Satorra-Bentler $(\mathrm{SB})^{19}$. Se evaluaron distintos escenarios para la búsqueda del modelo mejor ajustado, considerando la eliminación de ítems con cargas factoriales $<0,30, y$, mediante la inspección de los índices de modificación, la liberación de covarianzas de error para ítems altamente correlacionados. Los estadísticos de bondad de ajuste del modelo, con el método de $\mathrm{SB}$, fueron: (1) $\chi^{2}\left(\mathrm{SB} \chi^{2}\right) ;(2)$ índice de ajuste comparativo (CFI_SB), con un valor $\geq 0,90$ para un ajuste aceptable del modelo; (3) índice de Tucker-Lewis (TLI_SB), aplicando el mismo criterio que CFI para buen ajuste del modelo; (4) error cuadrático medio de aproximación (RMSEA_SB), con valores $\leq 0,05 \mathrm{y} \geq 0,10$ para un buen y pobre ajuste del modelo, respectivamente. Se examinó la correlación entre las escalas (covarianzas estandarizadas), y la consistencia interna de las escalas e ítems mediante el cálculo de los coeficientes $\alpha$ de Cronbach. Como prueba de validez de constructo convergente (i.e., prueba que dos medidas relacionadas teóricamente lo están empíricamente), se evaluó la asociación entre los puntajes en las escalas del CTQ-SF y marcadores de complejidad clínica de la depresión con la prueba no paramétrica U de Mann Whitney. De acuerdo con la robusta evidencia internacional sobre EAT y episodios depresivos clínicamente complejos en la edad adulta ${ }^{1}$, se hipotetizó que las medianas de los puntajes para las escalas del CTQSF serían superiores en pacientes con marcadores de complejidad clínica de la depresión vs. aquellos sin estos marcadores. Todos los análisis fueron asistidos por Stata $14.0^{19}$.

\section{Resultados}

Los estadísticos de bondad de ajuste para el modelo estructural de 25 ítems del CTQ-SF sugieren un grado de ajuste mediocre: $\mathrm{SB} \chi^{2}(265)=371,139$, valor de $\mathrm{p}=0,003$; RMSEA_SB $=0,067$; CFI_SB $=0,882 ; y$, TLI_SB $=0,866$. Este CFA preliminar detectó cargas factoriales muy bajas $(<0,30)$ para los ítems 1 y 6 en la escala NF. Con la excepción de estos ítems, se obtuvieron cargas factoriales $\geq 0,53$ (ítem 8 , escala $\mathrm{AE}$ ) en el resto de los ítems del CTQ-SF. Posteriormente, se reevaluó una estructura de cinco factores con 23 ítems (i.e., eliminando ítem 1 y 6 de NF), además de explorar la liberación de covarianzas de error entre los ítems 9 y 17 de EF, y 24 y 27 de AS. Con estas modificaciones se comprobó el modelo original de cinco dimensiones, con estadísticos de bondad de ajuste en rangos aceptables: $\mathrm{SB} \chi^{2}(218)=279,092$, valor $\mathrm{de} \mathrm{p}=0,003$; RMSEA_SB $=0,056$; CFI_SB $=0,930$; $\mathrm{y}$, TLI_SB $=0,919$. En este modelo todas las cargas factoriales fueron mayores a 0,40 , con la excepción del ítem $4(=0,38)$ en la escala de NF (Figura 1$)$. La mayor correlación entre las escalas se observó para el par NE y NF $(0,94)$.

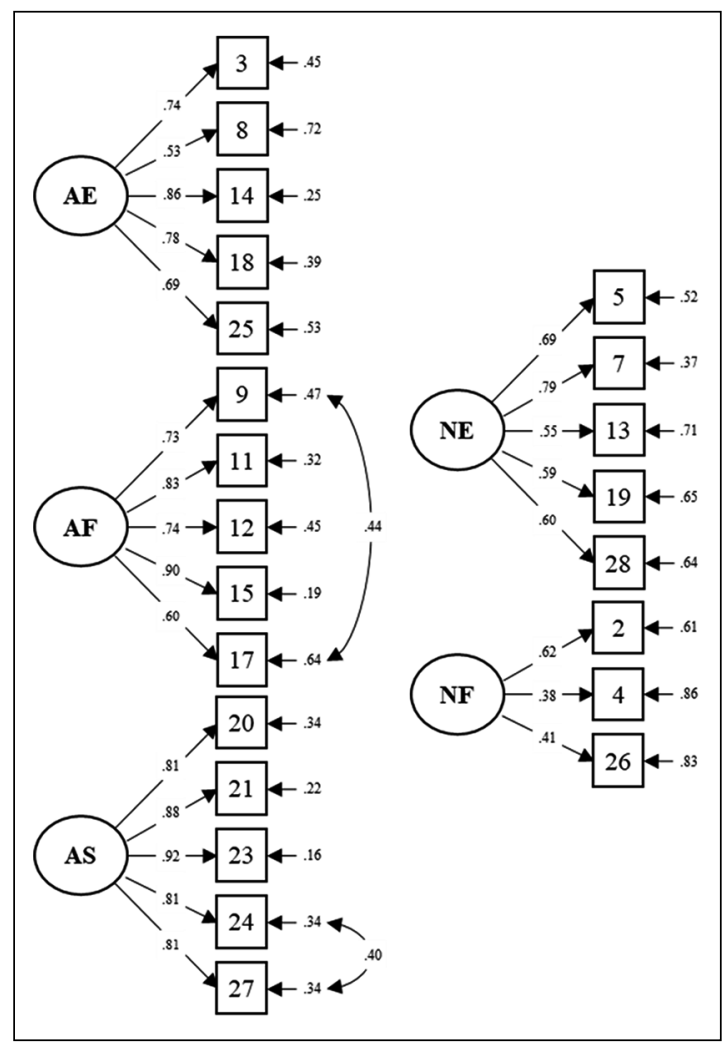

Figura 1. Cargas factoriales estandarizadas de los ítems de la versión chilena de CTQ-SF. Nota. AE = abuso emocional. $\mathrm{AF}=$ Abuso físico. $\mathrm{AS}=$ Abuso sexual. $\mathrm{NE}=$ Negligencia emocional. $\mathrm{NF}=$ Negligencia física. En los recuadros se indican los ítems de CTQ-SF, a la izquierda las cargas factoriales de cada ítem, y a la derecha los errores de las varianzas. Las líneas curvas al extremo derecho señalan las covarianzas del error. Todas las cargas factoriales se encuentran estandarizadas y son estadísticamente significativas $(p<0,001)$. 
Cabe señalar que la versión de 25 ítems tiene idéntica consistencia interna que la de 23 ítems $(\alpha$ de Cronbach $=0,89)$. Con excepción de la escala $\mathrm{NF}$, el resto de escalas exhiben consistencias internas en rangos adecuados a excelentes (Tabla 2).

La escala de AF fue la única que discriminó entre pacientes con depresión compleja vs no compleja $(Z=-2,644, p=0,008$, Tabla 3a), mien-

\section{Tabla 2. Confiabilidad de las escalas e ítems de la} versión chilena de CTQ-SF

\begin{tabular}{|c|c|c|}
\hline Variable & Datos & $\alpha$ \\
\hline Abuso emocional & $11,02(2,74)$ & 0,85 \\
\hline Ítem 3 & $2,29(1,23)$ & 0,80 \\
\hline Ítem 8 & $1,84(1,15)$ & 0,85 \\
\hline Ítem 14 & $2,21(1,16)$ & 0,79 \\
\hline Ítem 18 & $1,84(1,12)$ & 0,81 \\
\hline Ítem 25 & $2,83(1,45)$ & 0,82 \\
\hline Abuso físico & $7,48(3,88)$ & 0,87 \\
\hline Ítem 9 & $1,21(0,68)$ & 0,84 \\
\hline Ítem 11 & $1,54(1,01)$ & 0,83 \\
\hline Ítem 12 & $1,70(1,12)$ & 0,84 \\
\hline Ítem 15 & $1,85(1,20)$ & 0,82 \\
\hline Ítem 17 & $1,18(0,63)$ & 0,87 \\
\hline Abuso sexual & $8,10(5,16)$ & 0,93 \\
\hline Ítem 20 & $1,82(1,26)$ & 0,92 \\
\hline Ítem 21 & $1,37(1,08)$ & 0,91 \\
\hline Ítem 23 & $1,43(0,99)$ & 0,91 \\
\hline Ítem 24 & $1,79(1,25)$ & 0,91 \\
\hline Ítem 27 & $1,70(1,25)$ & 0,91 \\
\hline Negligencia emocional & $10,57(4,11)$ & 0,79 \\
\hline Ítem 5 (R) & $2,20(1,17)$ & 0,74 \\
\hline Ítem 7 (R) & $1,90(1,06)$ & 0,75 \\
\hline Ítem $13(R)$ & $2,13(1,08)$ & 0,76 \\
\hline Ítem 19 (R) & $2,24(1,10)$ & 0,76 \\
\hline Ítem 28 (R) & $2,10(1,16)$ & 0,76 \\
\hline Negligencia física & $4,61(1,89)$ & 0,41 \\
\hline Ítem 2 (R) & $1,78(1,03)$ & - \\
\hline Ítem 4 & $1,31(0,78)$ & 0,28 \\
\hline Ítem 26 (R) & $1,52(0,97)$ & 0,57 \\
\hline
\end{tabular}

Nota. Los datos son media (desviación estándar). Para ítem, $\alpha$ de la escala si se elimina el ítem. tras que todas las escalas del CTQ-SF lograron discriminar de una manera estadísticamente significativa entre pacientes con alto riesgo suicida o antecedentes de hospitalización psiquiátrica vs ninguno de estos antecedentes, exhibiendo la escala de NE las diferencias más grandes en este caso $(Z=-3,054, p=0,002$, Tabla $3 b)$.

\section{Discusión}

Los resultados principales de este estudio sugieren que la adaptación chilena del CTQ-SF presenta una aceptable adaptación al modelo de cinco factores, con propiedades psicométricas adecuadas. El análisis factorial confirmatorio (CFA) reveló que un mejor ajuste a esta estructura factorial se puede lograr eliminando dos ítems de la escala de negligencia física (NF) (ítems 1 y 6). Esta última escala es la menos fiable del instrumento. Por último, este estudio comprobó la validez convergente del CTQ-SF, ya que sus escalas fueron capaces de discriminar apropiadamente entre distintos marcadores de complejidad clínica de la depresión, particularmente en lo que respecta al grupo de pacientes con alto riesgo suicida y/o antecedentes de hospitalizaciones psiquiátricas previas.

En el presente estudio se comprobó la validez estructural del modelo original de cinco factores para el CTQ-SF${ }^{8}$, siendo consistente con los hallazgos de validaciones internacionales anteriores $^{9-11,13}$. La media de puntajes para las escalas del CTQ-SF fueron levemente inferiores a las señaladas en la validación española ${ }^{11}$, en una muestra clínica de mujeres que recibían tratamiento ambulatorio u hospitalario por problemas de salud mental. Estas diferencias, aunque menores, pueden ser atribuidas al mayor reporte de estrés adverso temprano (EAT) en mujeres versus hombres adultos, referida en distintos estudios epidemiológicos ${ }^{21,22}$. Más allá de estas discrepancias, la consistencia interna de las escalas del CTQ-SF, en rangos adecuados a excelentes, son muy similares a las observadas por Hernández et al. ${ }^{11}$ y Spinhoven et al. ${ }^{9}$, con excepción de la pobre consistencia interna de la escala de NF. Cabe destacar que estos y otros estudios $^{9-11,13}$, incluido el original ${ }^{8}$, han evidenciado el peor desempeño de esta última escala.

Relacionado con la heterogeneidad de la escala 
Tabla 3a. Puntuaciones en escalas de la versión chilena de CTQ-SF, estratificada por marcadores de complejidad clínica de la depresión: (1) depresión compleja

\begin{tabular}{|lcccc|}
\hline Escalas de CTQ-SF & $\begin{array}{c}\text { Total } \\
(\mathbf{n}=\mathbf{8 9})\end{array}$ & $\begin{array}{c}\text { Con marcador } \\
(\mathbf{n}=\mathbf{3 9 )}\end{array}$ & $\begin{array}{c}\text { Sin marcador } \\
(\mathbf{n}=\mathbf{5 0})\end{array}$ & U Mann Whitney \\
\hline Abuso emocional & $10(7-14)$ & $11(8-17)$ & $10(6-13)$ & $-1,72(0,086)$ \\
\hline Abuso físico & $6(5-8)$ & $7(5-9)$ & $5(5-7)$ & $-\mathbf{2 , 6 4}(\mathbf{0 , 0 0 8 )}$ \\
\hline Abuso sexual & $5(5-9)$ & $5(5-11)$ & $5,5(5-8)$ & $-0,51(0,610)$ \\
\hline Negligencia emocional & $11(7-13)$ & $12(7-15)$ & $10(7-12)$ & $-1,77(0,077)$ \\
\hline Negligencia física & $4(3-6)$ & $5(3-6)$ & $4(3-5)$ & $-1,72(0,085)$ \\
\hline
\end{tabular}

Nota. Los datos son mediana (percentil 25 - percentil 75). En U Mann Whitney, los datos son puntaje Z (valor de p), y, en negrita, se indican valores de $p$ estadísticamente significativos. La categoría "Con marcador" corresponde a los participantes con diagnóstico de depresión grave, o depresión con psicosis, alto riesgo suicida, o refractariedad, y "Sin marcador" corresponde a los participantes con diagnóstico de depresión leve o moderada.

\section{Tabla 3b. Puntuaciones en escalas de la versión chilena de CTQ-SF, estratificada por marcadores de complejidad clínica de la depresión: (2) alto riesgo suicida (MINI) y/o antecedentes de hospitalizaciones psiquiátricas}

\begin{tabular}{|c|c|c|c|c|}
\hline Escalas de CTQ-SF & $\begin{array}{c}\text { Total } \\
(n=89)\end{array}$ & $\begin{array}{l}\text { Con marcador } \\
(n=10)\end{array}$ & $\begin{array}{c}\text { Sin marcador } \\
(n=79)\end{array}$ & U Mann Whitney \\
\hline Abuso emocional & $10(7-13)$ & $14,5(9-18)$ & $10(7-13)$ & $-2,68(0,007)$ \\
\hline Abuso físico & $6(5-8)$ & $7(6-11)$ & $6(5-8)$ & $-2,25(0,025)$ \\
\hline Abuso sexual & $5(5-8)$ & $7,5(5-13)$ & $5(5-7)$ & $-2,94(0,003)$ \\
\hline Negligencia emocional & $10(6,5-13)$ & $12(10-17)$ & $10(6-12)$ & $-3,05(0,002)$ \\
\hline Negligencia física & $6(5-9)$ & $5,5(4-6)$ & $4(3-5)$ & $-2,67(0,008)$ \\
\hline
\end{tabular}

Nota. Los datos son mediana (percentil 25 - percentil 75). En U Mann Whitney, los datos son puntaje $Z$ (valor de p), y, en negrita, se indican valores de p estadísticamente significativos. La categoría "Con marcador" corresponde a los participantes con alto riesgo suicida según la MINI y/o antecedentes de hospitalizaciones psiquiátricas, y "Sin marcador" corresponde a los participantes que no tenían estos antecedentes.

de NF, Gerdner et al. ${ }^{10}$, apunta a la imprecisión teórica del constructo de negligencia física, sugiriendo que estaría compuesta por dos subdimensiones (falta de cuidado: ítems 2 y 26; y, falta de supervisión: ítems 1, 4, y 6), la primera de estas altamente correlacionada con la escala de negligencia emocional (NE). Lo anterior encontraría sustento empírico en la adaptación chilena del CTQ-SF, ya que tras eliminar los ítems 1 y 6, la correlación entre las escalas NE y NF incrementa sustancialmente desde 0,70 a 0,94 , junto con lograr un mejor ajuste de la estructura factorial general. La eliminación de ítems del CTQ-SF es un recurso que ha sido utilizado en otros estudios ${ }^{9,13}$, aunque es preferible la aplicación de la versión completa del mismo instrumento para facilitar la comparación entre resultados.
Este es el primer estudio en demostrar la validez convergente del CTQ-SF mediante el uso de marcadores de complejidad clínica de la depresión, consistente con un estudio holandés que determinó que mayores puntajes en el CTQSF se asociaban a mayor psicopatología depresiva y/o ansiosa ${ }^{9}$. Estos hallazgos se alinean con la evidencia provista por meta-análisis de estudios observacionales y experimentales que han documentado la estrecha asociación entre exposición a EAT y una presentación clínica compleja de la depresión en la adultez ${ }^{23,24} \mathrm{y}$ apoyan los resultados de un estudio chileno que encontró correlaciones estadísticamente significativas entre el EAT con mayor gravedad de la depresión y riesgo suicida, en una muestra de pacientes deprimidos en atención primaria de salud ${ }^{3}$. 
El presente estudio se realizó en una muestra de población consultante ambulatoria con depresión clínica. Futuros estudios debieran incorporar sujetos de poblaciones diversas, seleccionados probabilísticamente (i.e., clínicas y no clínicas), mayores tamaños muestrales y resguardar la paridad de género. Este tipo de condiciones pueden garantizar la evaluación de la invarianza estructural y de medición, para asegurar que las propiedades psicométricas son estables entre distintos grupos poblacionales ${ }^{9}$. Complementariamente, $y$ en acuerdo con otros estudios de validación del CTQ-SF ${ }^{9-11,13}$, la adaptación chilena de este instrumento no consideró los ítems 10, 16 y 22, que evalúan el subregistro de EAT. Futuros estudios debieran considerar la escala completa del CTQSF en sus análisis, puesto que la minimización del EAT es común ${ }^{25}$.

En resumen, la adaptación chilena del CTQ-SF en una muestra de pacientes con depresión clínica, cuenta con evidencia de validez estructural, confiabilidad y validez discriminante, constituyéndose en un instrumento con adecuadas propiedades psicométricas para la pesquisa y caracterización del EAT en Chile. Es de interés hacer notar que la mayor parte de los estudios locales han utilizado para la identificación del EAT la Escala de Trauma de Marshall ${ }^{3,26-27}$; este es un instrumento breve de siete ítems dicotómicos ${ }^{28}$, apto para un tamizaje inicial, por lo que el uso del CTQ-SF puede ser un complemento promisorio para una exploración más detallada del EAT. Previa autorización y licenciamiento, este estudio pone a disposición de clínicos e investigadores en Chile un instrumento considerado el estándar de referencia internacional en la evaluación retrospectiva del EAT, antecedente que ha sido relacionado consistentemente con mayor incidencia de enfermedades físicas y mentales del adulto ${ }^{1,2,4}$, con la consiguiente oportunidad de establecer protocolos clínicos de identificación temprana y tratamientos individualizados con mayor potencial de éxito.

\section{Derechos sobre el instrumento}

Reconocemos que los derechos del "Cuestionario de trauma infantil: un autoinforme retrospectivo (CTQ)" pertenecen exclusivamente a NCS Pearson y su uso está regulado y requiere del permiso y/o licencia de NCS Pearson Copyright ${ }^{\circ}$
1998 por NCS Pearson, INC. Todos los derechos reservados.

\section{Referencias}

1. Nemeroff CB. Paradise lost: the neurobiological and clinical consequences of child abuse and neglect. Neuron 2016; 89: 892-909.

2. Felitti VJ, Anda RF, Nordenberg D, Williamson DF, Spitz AM, Edwards V, et al. Relationship of childhood abuse and household dysfunction to many of the leading causes of death in adults. The Adverse Childhood Experiences (ACE) Study. Am J Prev Med 1998; 14: 245-58.

3. Vitriol V, Cancino A, Leiva-Bianchi M, Serrano C, Ballesteros S, Pothoff S, et al. Depresión adulta y experiencias infantiles adversas: evidencia de un subtipo depresivo complejo en consultantes de la atención primaria en Chile. Rev Med Chile 2017; 145: 1145-53.

4. Bellis MA, Hughes K, Ford K, Ramos Rodríguez G, Sethi $\mathrm{D}$, Passmore J. Life course health consequences and associated annual costs of adverse childhood experiences across Europe and North America: a systematic review and meta-analysis. Lancet Public Health 2019; 4: e51728.

5. Becker-Blease KA, Freyd JJ. Research participants telling the truth about their lives: the ethics of asking and not asking about abuse. Am Psychol 2006; 61: 218-26.

6. Edwards VJ, Dube SR, Felitti VJ, Anda RF. It's ok to ask about past abuse. Am Psychol 2007; 62: 327-8.

7. Saini SM, Hoffmann CR, Pantelis C, Everall IP, Bousman CA. Systematic review and critical appraisal of child abuse measurement instruments. Psychiatry Res 2019; 272: 106-13.

8. Bernstein DP, Stein JA, Newcomb MD, Walker E, Pogge D, Ahluvalia T, et al. Development and validation of a brief screening version of the Childhood Trauma Questionnaire. Child Abuse Negl 2003; 27: 169-90.

9. Spinhoven P, Penninx BW, Hickendorff M, van Hemert AM, Bernstein DP, Elzinga BM. Childhood Trauma Questionnaire: factor structure, measurement invariance, and validity across emotional disorders. Psychol Assess 2014; 26: 717-29.

10. Gerdner A, Allgulander C. Psychometric properties of the Swedish version of the Childhood Trauma Questionnaire-Short Form (CTQ-SF). Nord J Psychiatry 2009; 63: 160-70.

11. Hernández A, Gallardo-Pujol D, Pereda N, Arntz A, Bernstein DP, Gaviria AM, et al. Initial validation of the Spanish Childhood Trauma Questionnaire-Short Form: factor structure, reliability and association with 
parenting. J Interpers Violence 2013; 28: 1498-518.

12. Bernstein DP, Fink L, Handelsman L, Foote J, Lovejoy $\mathrm{M}$, Wensel $\mathrm{K}$, et al. Initial reliability and validity of a new retrospective measure of child abuse and neglect. Am J Psychiatry 1994; 151: 1132-6.

13. Thombs BD, Bernstein DP, Lobbestael J, Arntz A. A validation study of the Dutch Childhood Trauma Questionnaire-Short Form: factor structure, reliability, and known-groups validity. Child Abuse Negl 2009; 33: 518-23.

14. Ministerio de Salud de Chile. Guía clínica depresión en personas de 15 años y más. MINSAL: Santiago; 2013.

15. Ministerio de Salud de Chile. Listado de prestaciones específico. Régimen de Garantías Explícitas en Salud. Anexo Decreto Supremo No3, de 2016. MINSAL: Santiago; 2016. En: https://diprece.minsal.cl/wrdprss_minsal/ wp-content/uploads/2018/03/Lep_incluye-Decreto-8-de-2018.pdf.

16. National Institute for Health and Care Excellence. Depression in adults: recognition and management. NICE: London; 2009.

17. Bobes J, González MP, Sáiz PA, Bousoño M, Iglesias C, Bascarán MT, et al. La MINI (Mini International Neuropsychiatric Interview): una familia de entrevistas de ayuda diagnóstica en Psiquiatría y Atención Primaria, En: Gutiérrez M, Ezcurra J, González A, Pichot P (Eds.). Psiquiatría y otras especialidades médicas. Grupo Aula Médica: Madrid; 1998, pp. 401-19.

18. Ministerio de Salud. Programa nacional de prevención del suicidio: orientaciones para su implementación. MINSAL: Santiago; 2013. En: https://www.minsal.cl/ sites/default/files/Programa_Nacional_Prevencion.pdf.

19. Satorra A, Bentler PM. Corrections to test statistics and standard errors in covariance structure analysis. En: von Eye A, Clogg CC (Eds.). Latent variables analysis: applications for developmental research. SAGE: Thousand
Oaks, CA; 1994, pp. 399-419.

20. StataCorp. Stata Statistical Software: Release 14. College Station, TX: StataCorp LP; 2015.

21. Finkelhor D, Turner H, Ormrod R, Hamby SL. Violence, abuse, and crime exposure in a national sample of children and youth. Pediatrics 2009; 124: 1411-23.

22. Witt A, Brown RC, Plener PL, Brähler E, Fegert JM. Child maltreatment in Germany: prevalence rates in the general population. Child Adolesc Psychiatry Ment Health 2017; 11: 47.

23. Nanni V, Uher R, Danese A. Childhood maltreatment predicts unfavorable course of illness and treatment outcome in depression: a meta-analysis. Am J Psychiatry 2012; 169: 141-51.

24. Nelson J, Klumparendt A, Doebler P, Ehring T. Childhood maltreatment and characteristics of adult depression: meta-analysis. Br J Psychiatry 2017; 210: 96-104.

25. MacDonald K, Thomas ML, Sciolla AF, Schneider B, Pappas K, Bleijenberg G. Minimization of childhood maltreatment is common and consequential: results from a large, multinational sample using the Childhood Trauma Questionnaire. PLoS ONE 2016; 11: e0146058.

26. Cuneo C, González I, Jara M, Palomares L, Rammasy C, Cruz C. Validación externa de la escala de trauma de Marshall. En: Florenzano R, Weil P, Carvajal C, Cruz C (Eds.). Trauma infanto-juvenil y psicopatología adulta. Editorial Corporación de Promoción Universitaria: Santiago; 2005.

27. Weil K, Florenzano R, Vitriol V, Cruz C, Carvajal C, Fullerton C, et al. Trauma infantojuvenil y psicopatología adulta: un estudio empírico. Rev Med Chile 2004; 132: 1499-504.

28. Marshall RD, Schneier FR, Lin S-H, Simpson HB, Vermes D, Liebowitz M. Childhood trauma and dissociative symptoms in panic disorder. Am J Psychiatry 2000; 157: 451-3. 